\title{
Nonlinear modelling and dynamic stability analysis of a flexible Cartesian robotic manipulator with base disturbance and terminal load
}

\author{
Jinyong Ju, Wei Li, Mengbao Fan, Yuqiao Wang, and Xuefeng Yang \\ School of Mechatronic Engineering, China University of Mining and Technology, Xuzhou 221116, P.R. China \\ Correspondence to: Wei Li (cmeecumt512@yahoo.com)
}

Received: 5 December 2016 - Revised: 29 March 2017 - Accepted: 29 June 2017 - Published: 26 July 2017

\begin{abstract}
The flexible Cartesian robotic manipulator (FCRM) is coming into widespread application in industry. Because of the feeble rigidity and heavy deflection, the dynamic characteristics of the FCRM are easily influenced by external disturbances which mainly concentrate in the driving end and the load end. Thus, with the influence of driving base disturbance and terminal load considered, the motion differential equations of the FCRM under the plane motion of the base are constructed, which contain the forced and non-linear parametric excitations originated from the disturbances of base lateral and axial motion respectively. Considering the relationship between the coefficients of the motion differential equations and the mode shapes of the flexible manipulator, the analytic expressions of the mode shapes with terminal load are deduced. Then, based on multiple scales method and rectangular coordinate transformation, the average equations of the FCRM are derived to analyze the influence mechanism of base disturbance and terminal load on the system parametric vibration stability. The results show that terminal load mainly affects the node locations of mode shapes and mode frequencies of the FCRM, and the axial motion disturbance of the driving base introduces parametric excitation while the lateral motion disturbance generates forced excitation for the transverse vibration model of the FCRM. Furthermore, with the increase of the base excitation acceleration and terminal load, the parametric vibration instability region of the FCRM increases significantly. This study will be helpful for the dynamic characteristics analysis and vibration control of the FCRM.
\end{abstract}

\section{Introduction}

Cartesian coordinate robot is a common structure of industrial robots, which has been widely applied in handling operation, surface mount technology (SMT), welding operation and CNC machine (Park et al., 2013; Wu and Zhang, 2014; Srinivasan et al., 2015; Qian et al., 2016). With the development of modern machinery equipment to integration and lightweight, the flexible Cartesian robotic manipulator (FCRM) has received increasing attention. Compared with rigid structure, the FCRM has many advantages such as low energy consumption and heavy load. However, due to the low mode frequency and structure damping, the FCRM easily exhibits elastic vibration during the operation process, which has a heavy influence on the positioning accuracy and service life of the whole system, especially in high-speed oper- ations (Neto et al., 2013). Thus, the dynamic characteristics and vibration control of the FCRM should be further analyzed (Feliu et al., 2014; Kiang et al., 2015).

The FCRM system is composed of driving unit, flexible manipulator, and end effector, which is a typical multicoupling system. And the flexible manipulator can be assumed as a flexible beam to describe its dynamic characteristics (Mann et al., 2014). The calculated results by Zhang et al. (1995) showed that the results of the traditional modelling method were divergent when the base rotation frequency of the slender beam reached or exceeded the fundamental frequency of the beam. The origin of this phenomenon was that the rigid-flexible coupling effect of the flexible beam caused the total stiffness of the traditional zero-order approximation system model less than zero under the high-speed motion of the base (Feng and Hu, 2002; Duc et al., 2016). Then, with 
the modern robot technology developing to high-speed, the high-order coupling terms between the large overall motion of the base and the elastic vibration of the flexible manipulator for the modelling of the FCRM should be taken into consideration. On this basis, it is obvious that the base motion has a significant impact on the dynamic response of the FCRM.

Unfortunately, most of the existing literatures mainly analyze the parameters of the FCRM itself on its dynamic character by assuming that the output speed or torque of the driving unit are constant. Qiu (2012) studied the nonlinear vibration control for a Cartesian flexible manipulator by ignoring the torque ripple of the driving motor. The trajectory planning of the base was applied for the vibration control of a ?exible Cartesian robot manipulator with the assumption that the base motion can completely in accordance with the specified manner (Abe, 2011). The eigenspectrum and eigenfunctions of a flexible manipulator are investigated by ignoring the disturbance of the base movement (Coleman and Mcsweeney, 2004). However, considering the coupling fact of the flexible manipulator and the driving base, there are some inadequacies for the dynamic analysis of the flexible manipulator in existing research. Taking the motor drives as an example, it is difficult for the driving base to move with uniform velocity or constant axial force under the influence of power harmonics, motor rotor eccentricity and mechanism gap.

With the fluctuation components of the transmission velocity or axial force of the driving base considered, the FCRM may appear large amplitude vibration of parametric excitation and present complex dynamical characteristics. Atsumi et al. (2005) analyzed the influence of the transmission error on the servo accuracy and the results indicated that the high frequency components had a significant effect. Liu et al. (2015) derived the analytical solutions of a harmonicdisturbance exciting system for a translational flexible-link manipulator. Accordingly, the influence of base disturbance and other external factors on the dynamic characteristics of the flexible manipulator is obvious. With the development of nonlinear dynamics theory, the vibration mechanism of the flexible manipulator has been deeply understood. Based on Lie group theory, Özkaya and Pakdemirli (2002) analyzed the vibration stability of an Euler beam with timevarying velocity motion. By multiple scales method, Chen and Yang (2005) investigated the dynamic stability problems of an axially accelerating beam under the conditions of combination and principal parametric resonance. Similarly, with an added mass placed at an intermediate position, Pratiher and Bhowmick (2011) investigated the nonlinear dynamic characteristics of a Cartesian manipulator. De Luca and Siciliano (1991) analyzed the influences of the payload on the first-order mode shapes of the link-1 and link-2 for a planar multilink lightweight robot, but this research did not further study the impact of the payload on the system dynamics stability. Through the summary of the existing literature, it is obvious that the model parameters of the flexible ma-

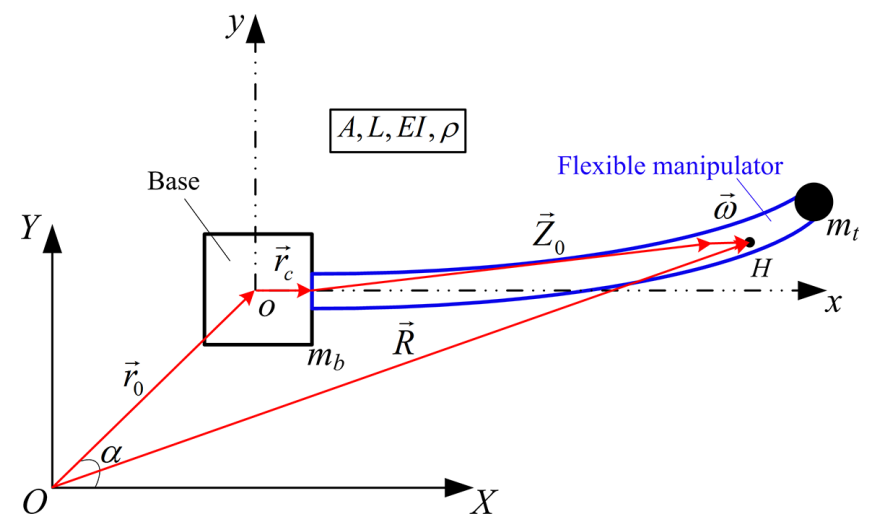

Figure 1. Schematic diagram of the FCRM system under large overall motion.

nipulator are closely related to its mode shapes. However, there is very little research about considering the influence of terminal load on the mode shapes, especially the highorder modes, of the flexible manipulator before the dynamics stability analysis, which may cause dynamic instability phenomenon and unstable response. The FCRM is designed to operate the appropriate objects. Due to the structure characteristics, the terminal load, especially the variable load conditions, can significantly affect the mode frequency and vibration mode of the flexible manipulator. Thus, during the nonlinear dynamics and stability analysis of the FCRM, the impact of terminal load should be taken into consideration firstly.

The objectives of this paper are to construct the nonlinear dynamics model of the FCRM with combining the highorder coupling terms between the large overall motion of the driving base and the elastic vibration of the flexible manipulator. Considering the influence of terminal load on the boundary conditions, the mode shapes of the flexible manipulator are deduced. Based on multiple scales method, the impact mechanisms of terminal load and driving base disturbance on the nonlinear dynamics and vibration stability of the FCRM are analyzed. The structure of this paper is organized as follows. The nonlinear dynamic modelling of the FCRM is given in Sect. 2. Section 3 analyzes the influence of terminal load on the mode shapes of the flexible manipulator. Section 4 provides the nonlinear response analysis of the FCRM, including the amplitude-frequency characteristics and parametric vibration stability of the FCRM. Finally, conclusions are drawn in Sect. 5.

\section{Nonlinear dynamic modelling}

The structural diagram of the FCRM is shown in Fig. 1, where $O X Y$ denotes inertial coordinate system and oxy denotes follow-up coordinate system moving with the FCRM system. The motion range of the driving base is $0.8 \mathrm{~m} \times 1 \mathrm{~m}$, which is large overall motion relative to the elastic vibration 
displacement of the flexible manipulator. The flexible manipulator undergoing large overall $O X Y$-plane motion is fastened to the driving base, which is driven by a permanent magnet $\mathrm{AC}$ servomotor and screw-nut pairs. It is necessary for dynamics modelling to assume that: (1) The flexible manipulator satisfies the Euler-Bernoulli beam assumptions; (2) for the flexible manipulator, the material is homogeneous and the areas of the cross sections are equal; (3) sections of the flexible manipulator perpendicular to the axis that are plane before deformation remain plane after deformation, and the shear and torsion effect perpendicular to the axis are neglected; (4) under large overall motion, the flexible manipulator occurs medium and small deformation and the axial deformation, relative to the lateral deformation, is small deformation; (5) The connection between the base and the flexible manipulator is pure rigid.

Only the tensile and bending deformations of the FCRM are considered. Then, according to deformation geometry condition, one can obtain:

$x+\omega_{u}=\int_{0}^{x} \sqrt{\left(1+\frac{\mathrm{d} \omega_{x}}{\mathrm{~d} \xi}\right)^{2}+\left(\frac{\mathrm{d} \omega_{y}}{\mathrm{~d} \xi}\right)} \mathrm{d} \xi$.

Spreading Eq. (1) by Taylor series and keeping to $\mathrm{O}\left(\mathrm{d} \omega_{x} / \mathrm{d} x\right)$ and $\mathrm{O}\left(\left(\mathrm{d} \omega_{y} / \mathrm{d} x\right)^{2}\right)$ items (Zi and Zhou, 2016), one can obtain:

$$
\begin{aligned}
\omega_{u} & =\omega_{x}+\omega_{\mathrm{g}} \\
& =\omega_{x}+\frac{1}{2} \int_{0}^{x}\left(\frac{\mathrm{d} \omega_{y}}{\mathrm{~d} \sigma}\right)^{2} \mathrm{~d} \sigma .
\end{aligned}
$$

The axial force and lateral bending moment of the flexible manipulator can be expressed as:

$$
\begin{aligned}
& P=E A \frac{\partial \omega_{u}}{\partial x}, \\
& M=E I \frac{\partial^{2} \omega_{y}}{\partial x^{2}}\left(1-2 \frac{\partial \omega_{u}}{\partial x}+\frac{1}{2}\left(\frac{\partial \omega_{y}}{\partial x}\right)^{2}\right)-E I \frac{\partial^{2} \omega_{u}}{\partial x^{2}} \frac{\partial \omega_{y}}{\partial x} .
\end{aligned}
$$

Then, the elastic potential energy of the FCRM system can be further shown as:

$U=\int_{0}^{L} \frac{P^{2}}{2 E A} \mathrm{~d} x+\int_{0}^{L} \frac{M^{2}}{2 E I} \mathrm{~d} x$.

Under large overall plane motion, the radius vector after deformation of $H(x, y)$ on the flexible manipulator can be written as:

$\boldsymbol{R}=\boldsymbol{r}_{\mathrm{o}}+\boldsymbol{r}_{\mathrm{c}}+z_{\mathrm{o}}+\boldsymbol{\omega}$,

where, $\boldsymbol{r}_{0}=\left(\boldsymbol{R}_{x}, \boldsymbol{R}_{y}\right)^{T}$ is the radius vector of the base centroid with respect to the inertial coordinate $O X Y, \boldsymbol{r}_{\mathrm{c}}=(a$, $0)^{T}$ is the radius vector of the base centroid with respect to the initial deformation position of the flexible manipulator, $z_{0}=(x, y)^{T}$ is the radius vector of $H(x, y)$ with respect to the follow-up coordinate oxy before deformation and $\omega=\left(\omega_{u}, \omega_{y}\right)^{T}$ is the deformation displacement vector of $H(x, y)$.

Differentiating Eq. (6) with respect to time yield, the speed vector of $H(x, y)$ can be expressed as:

$\dot{\boldsymbol{R}}=\dot{\boldsymbol{r}}_{\mathrm{o}}+\dot{\boldsymbol{\omega}}$

Moreover, the radius vector of the terminal operated object can be written as:

$\boldsymbol{R}_{m_{\mathrm{t}}}=\boldsymbol{r}_{\mathrm{o}}+\boldsymbol{r}_{\mathrm{c}}+\boldsymbol{z}_{m_{\mathrm{t}}}+\boldsymbol{\omega}_{m_{\mathrm{t}}}$

where, $z_{m_{\mathrm{t}}}=(L, 0)^{T}$ is the radius vector of the terminal operated object with respect to the follow-up coordinate oxy before deformation and $\omega_{m_{\mathrm{t}}}=\left(\omega_{u}(L, t), \omega_{y}(L, t)\right)^{T}$ is the deformation displacement vector.

Differentiating Eq. (8) with respect to time yield, the speed vector of the terminal operated object can be shown as:

$\dot{\boldsymbol{R}}_{m_{\mathrm{t}}}=\dot{\boldsymbol{r}}_{\mathrm{o}}+\dot{\boldsymbol{\omega}}_{m_{\mathrm{t}}}$.

Then, the kinetic energy of the FCRM system can be expressed as:

$T=\frac{1}{2} \int{ }_{V} \rho \dot{\boldsymbol{R}}^{T} \dot{\boldsymbol{R}} \mathrm{d} V+\frac{1}{2} m_{\mathrm{b}} \dot{\boldsymbol{r}}_{\mathrm{o}}^{2}+\frac{1}{2} m_{\mathrm{t}} \dot{\boldsymbol{R}}_{m_{\mathrm{t}}}^{T} \dot{\boldsymbol{R}}_{m_{\mathrm{t}}}$.

Based on Rayleigh-Ritz method (Huang and Li, 2010), the longitudinal and transverse vibration of the FCRM can be obtained as:

$\omega_{x}(x, t)=\boldsymbol{\psi}(x) \boldsymbol{Q}(t)=\sum_{i=1}^{N_{1}} \psi_{i}(x) Q_{i}(t)$,

$\omega_{y}(x, t)=\boldsymbol{\Phi}(x) \boldsymbol{q}(t)=\sum_{i=1}^{N_{2}} \phi_{i}(x) q_{i}(t)$.

The second Lagrange equation expression can be written as:

$\frac{\mathrm{d}}{\mathrm{d} t}\left(\frac{\partial T}{\partial \dot{\vartheta}}\right)-\frac{\partial T}{\partial \vartheta}+\frac{\partial U}{\partial \vartheta}=F_{\vartheta}$.

By substituting Eqs. (1) and (10)-(12) into Eq. (13), the coupling dynamic model of the longitudinal and transverse 
vibration for the FCRM system can be deduced as:

$$
\begin{aligned}
& \left(A \int_{0}^{L} \rho \psi_{k} \psi_{k}^{T} \mathrm{~d} x+m_{\mathrm{t}} \psi_{k}^{2}(L)\right) \ddot{Q}_{k}+\int_{0}^{L} E A \psi_{k, x} \psi_{k, x}^{T} \mathrm{~d} x Q_{k} \\
& -\sum_{i=1}^{N_{2}} \sum_{j=1}^{N_{2}}\left(A \int_{0}^{L} \rho \psi_{k} \alpha_{i j} \mathrm{~d} x+m_{\mathrm{t}} \psi_{k}(L) \beta_{i j}\right)\left(\dot{q}_{i} \dot{q}_{j}+q_{i} \ddot{q}_{j}\right) \\
& +\left(A \int_{0}^{L} \rho \psi_{k} \mathrm{~d} x+\frac{1}{2} m_{\mathrm{t}} \psi_{k}(L)\right) \ddot{R}_{x}=0 \\
& \quad-A \rho \sum_{i=1}^{N_{2}}\left(\int_{0}^{L} \alpha_{i k} \mathrm{~d} x+m_{\mathrm{t}} \beta_{i k}\right) q_{i} \ddot{R}_{x} \\
& +\left(A \rho \int_{0}^{L} \phi_{k} \mathrm{~d} x+\frac{1}{2} m_{\mathrm{t}} \phi_{k}(L)\right) \ddot{R}_{y} \\
& +\left(A \rho \int_{0}^{L} \phi_{k} \phi_{k}^{T} \mathrm{~d} x+m_{\mathrm{t}} \phi_{k}^{2}(L)\right) \ddot{q}_{k}+E I \int_{0}^{L} \phi_{k, x x} \mathrm{~d} x q_{k} \\
& +A \rho \sum_{i=1}^{N_{2}} \sum_{j=1}^{N_{2}} \sum_{h=1}^{N_{2}}\left(\int_{0}^{L} \alpha_{i j} \alpha_{h k} \mathrm{~d} x+\beta_{i j} \beta_{h k}\right) q_{i}\left(q_{j} \ddot{q}_{h}+\dot{q}_{j} \dot{q}_{h}\right) \\
& +\sum_{i=1}^{N_{2}} \sum_{j=1}^{N_{2}} \sum_{h=1}^{N_{2}} E I\left(v_{j k} \beta_{i h}+v_{i k} \beta_{j h}\right) q_{i} q_{j} q_{h}=0 \\
& \quad k=1,2, \ldots N_{2},
\end{aligned}
$$

where, $\quad(*)_{i, x}$ shows $(*)_{i} / \mathrm{d} x, \quad \alpha_{i j}=\int_{0}^{x} \phi_{i, \xi}(\xi) \phi_{j, \xi}^{T}(\xi) \mathrm{d} \xi$, $\beta_{i j}=\int_{0}^{L} \phi_{i, x} \phi_{j, x}^{T} \mathrm{~d} x$ and $v_{i j}=\int_{0}^{L} \phi_{i, x x}(x) \phi_{j, x x}^{T}(x) \mathrm{d} x$.

One may note that the coupling dynamic model of the FCRM system contains inertia $\left(A \rho \sum_{i=1}^{N_{2}} \sum_{j=1}^{N_{2}} \sum_{h=1}^{N_{2}}\left(\int_{0}^{L} \alpha_{i j} \alpha_{h k} \mathrm{~d} x+\beta_{i j} \beta_{h k}\right) q_{i}\left(q_{j} \ddot{q}_{h}+\dot{q}_{j} \dot{q}_{h}\right)\right)$ and cubic geometric $\left(\sum_{i=1}^{N_{2}} \sum_{j=1}^{N_{2}} \sum_{h=1}^{N_{2}} E I\left(v_{j k} \beta_{i h}+v_{i k} \beta_{j h}\right) q_{i} q_{j} q_{h}\right)$ nonlinear terms. From Eqs. (14) and (15), it is obtained that the axial and lateral motions of the driving base generate non-linear parametric excitation and forced excitation for the transverse vibration model of the FCRM, respectively. And the axial motion of the driving base introduces forced excitation for the longitudinal vibration model of the FCRM.

Because there is small deformation in the longitudinal vibration compared with the transverse vibration, the effect caused by the longitudinal vibration is not taken into consideration. Compared with the forced vibration, the parametric excitation is more complex and has a greater impact on the
FCRM system (Yan et al., 2012). Thus, the parametric excitation, caused by the axial movement disturbance of the base, of the FCRM system is investigated. To the FCRM system, the excitations of the unstable motion of the base, caused by the power harmonics, motor rotor eccentricity, mechanism gap and so on of the motor driving system, have obvious periodicity. Considering the periodic disturbance signals can be transformed into trigonometric functions by Fourier transform, for simplifying the analysis, the disturbance acceleration of the axial excitation is defined as $\ddot{R}_{x}=a_{0} \cos \Omega t$, where $a_{0}$ and $\Omega$ represent the amplitude and angular frequency.

The following dimensionless parameters are introduced for simplifying the coupling dynamic model:

$y_{k}=\frac{q_{i}}{L}, \tau=\frac{t}{T}, T=\sqrt{\frac{\rho A L^{4}}{E I}}, \eta=\frac{x}{L}, \bar{m}=\frac{m_{\mathrm{t}}}{m_{L}}=\frac{m_{\mathrm{t}}}{L A \rho}$.

Through substituting Eq. (16) into Eq. (15) and introducing the linear viscous damping and a book keeping parameter $\varepsilon$, Eq. (15) can be simplified as:

$$
\begin{aligned}
& \ddot{y}_{k}+\omega_{k}^{2} y_{k}+\varepsilon \\
& \left(\begin{array}{l}
2 \xi_{k} \dot{y}_{k}-\sum_{i=1}^{N_{2}}\left(C_{i}^{k}+C_{i}^{m_{\mathrm{t}}}\right) y_{i} \cos \bar{\omega} \tau+\sum_{i=1}^{N_{2}} \sum_{j=1}^{N_{2}} \sum_{h=1}^{N_{2}} D_{i j h}^{k} y_{i} y_{j} y_{h}+ \\
\sum_{i=1}^{N_{2}} \sum_{j=1}^{N_{2}} \sum_{h=1}^{N_{2}}\left(E_{i j h}^{k}+E_{i j h}^{m_{\mathrm{t}}}\right) y_{i}\left(y_{j} \ddot{y}_{h}+\dot{y}_{j} \dot{y}_{h}\right)
\end{array}\right) \\
& =0 \quad k=1,2, \ldots N_{2} \text {, }
\end{aligned}
$$

where, $\quad \bar{\omega}=\Omega T, \quad \omega_{k}^{2}=\frac{K_{k}}{M_{k}+M M_{k}}, \quad K_{k}=\int_{0}^{1} \phi_{k, \eta \eta}^{2} \mathrm{~d} \eta$, $C_{i}^{k}=\frac{a_{0} T^{2} \widetilde{C}_{i}^{k}}{\varepsilon L\left(M_{k}+M M_{k}\right)}, \quad \widetilde{C}_{i}^{k}=\int_{0}^{1} \widetilde{\alpha}_{i k} \mathrm{~d} \eta, \quad C_{i}^{m_{\mathrm{t}}}=\frac{\frac{1}{2} \bar{m} a_{0} T^{2} \widetilde{\beta}_{i k}}{\varepsilon L\left(M_{k}+M M_{k}\right)}$, $D_{i j h}^{k}=\frac{L^{6} T^{2}}{\varepsilon\left(A \rho M_{k}+M M_{k}\right)}\left(\widetilde{v}_{j k} \widetilde{\beta}_{i h}+\widetilde{v}_{i k} \widetilde{\beta}_{j h}\right), E_{i j h}^{k}=\frac{\widetilde{E}_{i j h}^{k}}{\varepsilon\left(M_{k}+M M_{k}\right)}$, $\widetilde{E}_{i j h}^{k}=\int_{0}^{1} \widetilde{\alpha}_{i j} \widetilde{\alpha}_{h k} \mathrm{~d} x, \quad E_{i j h}^{m_{\mathrm{t}}}=\frac{\bar{m} \int_{0}^{1} \phi_{i, \eta} \phi_{j, \eta} \phi_{h, \eta} \phi_{k, \eta} \mathrm{d} \eta}{\varepsilon L\left(M_{k}+M M_{k}\right)}, \quad M_{k}=$ $\int_{0}^{1} \phi_{k}^{2} \mathrm{~d} \eta, \quad M M_{k}=\bar{m} \phi_{k}^{2}(L), \quad \widetilde{\alpha}_{i k}=\int_{0}^{\mu} \phi_{i, \xi}(\xi) \phi_{j, \xi}^{T}(\xi) \mathrm{d} \xi$, $\widetilde{\beta}_{i j}=\int_{0}^{L} \phi_{i, x} \phi_{j, x}^{T} \mathrm{~d} x, \widetilde{v}_{i j}=\int_{0}^{1} \phi_{i, x x}(x) \phi_{j, x x}^{T}(x) \mathrm{d} x, 0 \leq \varepsilon \ll 1$.

\section{Mode shapes analysis for the FCRM system}

From Eq. (17), we can obtain that the associated parameters of the dynamic model for the transverse vibration of the FCRM system are mainly determined by the mode shapes of the flexible manipulator. Thus, the mode shapes under the influence of terminal load should be analyzed firstly (Chu et al., 2013). The structural parameters of the FCRM system are defined as: $L=0.4 \mathrm{~m}, \rho=2030 \mathrm{~kg} \mathrm{~m}^{-3}, A=1.35 e^{-4} \mathrm{~m}^{2}$ and $E=25 \mathrm{GPa}$. The flexible manipulator is assumed as an 

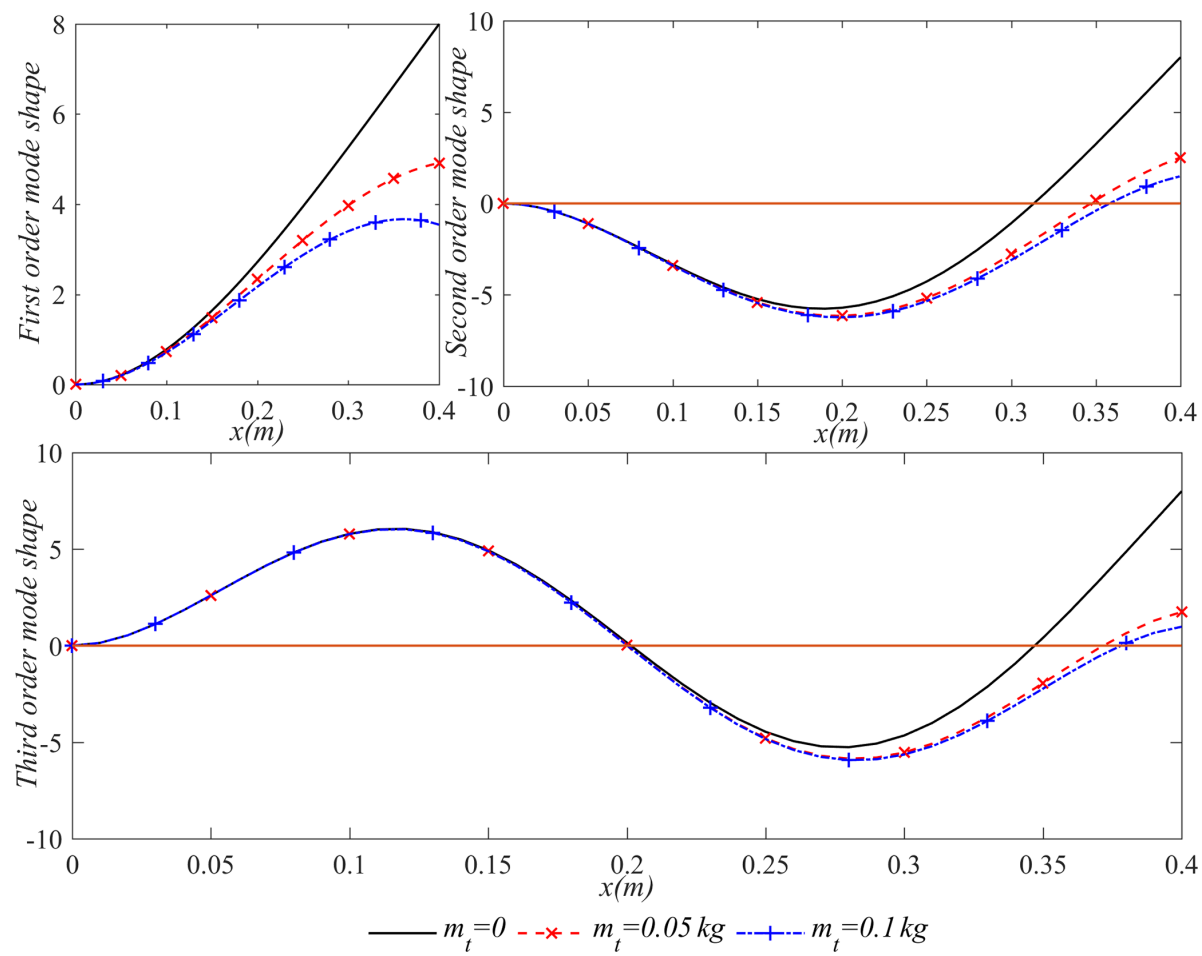

Figure 2. Contrast curves of the first three order mode shapes for the flexible manipulator with terminal load.

Euler-Bernoulli beam, whose standard form of mode shapes can be represented as:

$\phi_{k}(x)=\gamma_{1} \sin \beta_{k} x+\gamma_{2} \cos \beta_{k} x+\gamma_{3} \sinh \beta_{k} x+\gamma_{4} \cosh \beta_{k} x$,

where, $\gamma_{1}, \gamma_{2}, \gamma_{3}$ and $\gamma_{4}$ are constants which are determined by the boundary conditions.

Because the deflection and the angle of the fixed end for the flexible manipulator are equal to zero, the fixed end boundary conditions of the flexible manipulator can be expressed as:

$x=0:\left\{\begin{array}{l}\omega_{y}(0, t)=0 \\ \frac{\partial \omega_{y}(0, t)}{\partial x}=0\end{array}\right.$

Considering the influence of terminal load, the bending moment and shear force of the free end for the flexible manipulator are equal to zero and the inertia force of terminal load, respectively. Then, the free end boundary conditions of the flexible manipulator can be specified as:

$x=L:\left\{\begin{array}{l}E I \frac{\partial^{2} \omega_{y}(L, t)}{\partial x^{2}}=0 \\ \frac{\partial}{\partial x}\left(E I \frac{\partial^{2} \omega_{y}(L, t)}{\partial x^{2}}\right)=m_{\mathrm{t}} \frac{\partial^{2} \omega_{y}(L, t)}{\partial t^{2}}\end{array}\right.$.

Substituting Eq. (12) into Eqs. (19) and (20), the mode shapes of the flexible manipulator satisfy the following ex- pressions which are shown as:

$$
\begin{aligned}
& \left\{\begin{array}{l}
\phi_{k}(0)=0 \\
\frac{\mathrm{d} \phi_{k}(0)}{\mathrm{d} x}=0
\end{array}\right. \\
& \left\{\begin{array}{l}
E I \frac{\mathrm{d} \phi_{k}^{3}(L)}{\mathrm{d} x^{3}}=-m_{\mathrm{t}} \omega_{k}^{2} \phi_{k}(L) \\
E I \frac{\mathrm{d} \phi_{k}^{2}(L)}{\mathrm{d} x^{2}}=0
\end{array}\right.
\end{aligned}
$$

Substitution of Eq. (21) into Eq. (18) yields:

$$
\left\{\begin{array}{l}
\gamma_{1}+\gamma_{3}=0 \\
\gamma_{2}+\gamma_{4}=0
\end{array} .\right.
$$

By substituting Eq. (22) into Eq. (18), the result combined with Eq. (23) yields:

$\left[\begin{array}{ll}\Lambda_{11} & \Lambda_{12} \\ \Lambda_{21} & \Lambda_{22}\end{array}\right]\left[\begin{array}{l}\gamma_{1} \\ \gamma_{2}\end{array}\right]=0$. 

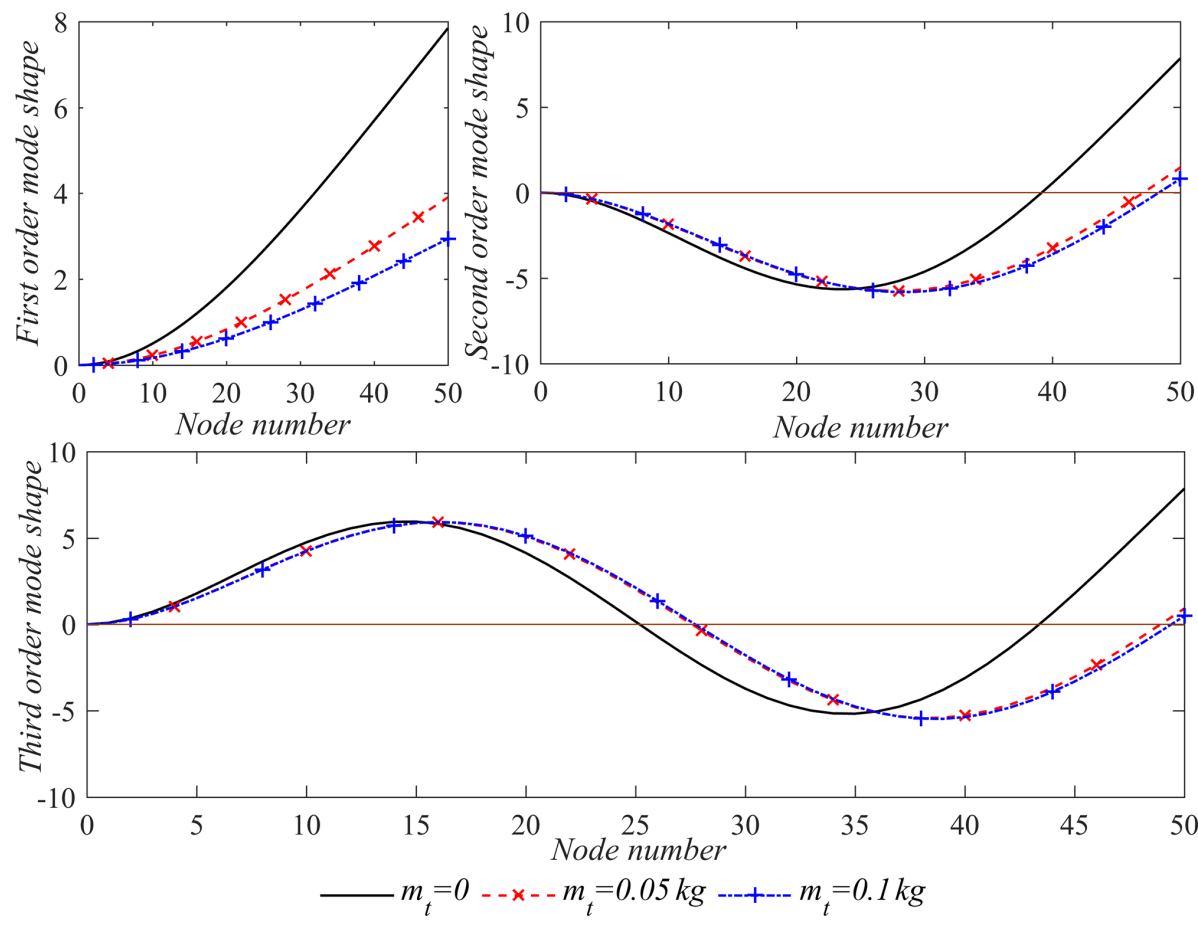

Figure 3. Contrast curves of the first three order mode shapes for the flexible manipulator with terminal load by FEM.

where, $\quad \Lambda_{11}=-\sin \beta_{k} L-\sinh \beta_{k} L ; \quad \Lambda_{12}=-\cos \beta_{k} L-$ $\cosh \beta_{k} L$;

$$
\begin{aligned}
\Lambda_{21} & =K_{m_{\mathrm{t}}} \sin \beta_{k} L-K_{m_{\mathrm{t}}} \sinh \beta_{k} L \\
& +\beta_{k}^{3} \cos \beta_{k} L+\beta_{k}^{3} \cosh \beta_{k} L ; \\
\Lambda_{22} & =K_{m_{\mathrm{t}}} \cos \beta_{k} L-K_{m_{\mathrm{t}}} \cosh \beta_{k} L-\beta_{k}^{3} \sin \beta_{k} L \\
& +\beta_{k}^{3} \sinh \beta_{k} L ; \quad K_{m_{\mathrm{t}}}=-\frac{m_{\mathrm{t}} \omega_{k}^{2}}{E I} .
\end{aligned}
$$

In order to guarantee the existence of nontrivial solution for $\gamma_{1}$ and $\gamma_{2}$, the determinant of the coefficient matrix of Eq. (24) should be equal to zero. Then, the frequency equation of the flexible manipulator with terminal load can be got as:

$$
\begin{gathered}
\bar{m} \beta_{k} L\left(\sinh \beta_{k} L \cos \beta_{k} L-\sin \beta_{k} L \cosh \beta_{k} L\right) \\
+\cos \beta_{k} L \cosh \beta_{k} L+1=0
\end{gathered}
$$

The relationship between $\gamma_{1}$ and $\gamma_{2}$ can be confirmed by Eqs. (23) and (24). Afterwards, the mode shapes of the flexible manipulator with terminal load can be specified as:

$\phi_{k}(x)=\left(\cos \beta_{k} x-\cosh \beta_{k} x\right)+\mu\left(\sin \beta_{k} x-\sinh \beta_{k} x\right)$,

where $\mu=-\frac{K_{m_{\mathrm{t}}} \cos \beta_{k} L-K_{m_{\mathrm{t}}} \cosh \beta_{k} L-\beta_{k}^{3} \sin \beta_{k} L+\beta_{k}^{3} \sinh \beta_{k} L}{K_{m_{\mathrm{t}}} \sin \beta_{k} L-K_{m_{\mathrm{t}}} \sinh \beta_{k} L+\beta_{k}^{3} \cos \beta_{k} L+\beta_{k}^{3} \cosh \beta_{k} L}$.
If $m_{\mathrm{t}}=0$, Eqs. (25) and (26) can be transformed as:

$$
\begin{aligned}
& \cos \beta_{k} L \cosh \beta_{k} L+1=0, \\
& \phi_{k}(x)=\left(\cos \beta_{k} x-\cosh \beta_{k} x\right) \\
&-\frac{\sinh \beta_{k} L-\sin \beta_{k} L}{\cos \beta_{k} L+\cosh \beta_{k} L}\left(\sin \beta_{k} x-\sinh \beta_{k} x\right) .
\end{aligned}
$$

Equations (27) and (28) are the frequency equations and mode shapes of the flexible manipulator without terminal load which is consistent with the expressions of a standard cantilever beam concluded by Singiresu (2004). Then, the validity of Eqs. (25) and (26) are verified.

Figure 2 shows the first three order mode shapes of the flexible manipulator with terminal load. It is obtained that the terminal load has an apparent effect on the mode shapes and there may cause error if the terminal load is ignored. With the increase of terminal load, it is seen that the amplitude of the first-order mode shape decreases obviously, and the second node of the second-order mode shape as well as the third node of the third-order mode shape move away with the fixed end of the flexible manipulator. Additionally, because the second node of the second mode shape and the third node of the third mode shape are already close to the free end of the flexible manipulator, the influence degree of terminal load on the second mode shape and third mode shape of the flexible manipulator will decrease with the increase of terminal load. Then, the relative vibration amplitude near the free end of the flexible manipulator reduces observably. In order to further verify the correctness of the deduced mode shapes functions, the modal analysis of the flexible manipulator with terminal 
Table 1. Mode frequencies of the first three order modes with terminal load by FEM.

\begin{tabular}{lccc}
\hline & \multicolumn{3}{c}{ Mode frequencies/Hz } \\
\cline { 2 - 4 } terminal load & First order mode & Second order mode & Third order mode \\
\hline$m_{\mathrm{t}}=0$ & 7.080 & 44.443 & 124.638 \\
$m_{\mathrm{t}}=0.05 \mathrm{~kg}$ & 3.479 & 33.199 & 103.314 \\
$m_{\mathrm{t}}=0.1 \mathrm{~kg}$ & 2.619 & 32.234 & 102.176 \\
\hline
\end{tabular}

load is implemented in ANSYS. Additionally, the analysis results by finite element method (FEM) in ANSYS are exported to describe more clearly and the results are shown in Fig. 3. Through comparing Figs. 2 and 3, it is obvious that the variation tendency of the first three mode shapes, obtained by theoretical analysis, is consistent with the results by ANSYS. Thus, the correctness of the deduced mode shape functions is verified.

Table 1 shows the mode frequencies of the first three order modes for the flexible manipulator with terminal load by FEM. It is known that the increase of terminal load reduces the mode frequencies of the flexible manipulator. Owing to the relationship between the mode frequency and the mode shape is same as the relationship between eigenvalue and eigenvector, the change of the mode frequency inevitably lead to the change of the mode shape. Moreover, by comparing the mode frequencies and mode shapes of the first two order modes, it is seen that the closer the node to the fixed end, the higher the mode frequency of the flexible manipulator. From this relationship, we can conclude that the existence of terminal load causes the decrease of the mode frequencies, and then leads to the change of the nodes locations of the mode shapes for the flexible manipulator. Thus, in the nonlinear response analysis of the FCRM system, the influence of terminal load on the mode shapes should be taken into consideration.

\section{Nonlinear response analysis}

Because Eq. (17) contains multiple nonlinear terms, the accurate analytical solution is hard to solve. Thus, based on multiple scales method, the approximate analytical solution for Eq. (17) is obtained which can be written as:

$y_{k}(\tau, \varepsilon)=y_{k 0}\left(T_{0}, T_{1}\right)+\varepsilon y_{k 1}\left(T_{0}, T_{1}\right)$,

where $T_{0}=\tau$ and $T_{1}=\varepsilon \tau$.
Substituting Eq. (29) into Eq. (17) and equating the coefficients of $\varepsilon^{0}$ and $\varepsilon^{1}$ lead to:

$$
\begin{aligned}
& \text { order } \varepsilon^{0}: D_{0}^{2} y_{k 0}+\omega_{k}^{2} y_{k 0}=0, \\
& \text { order } \varepsilon^{1}: D_{0}^{2} y_{k 1}+\omega_{k}^{2} y_{k 1}+2 \xi_{k} D_{0} y_{k 0}+2 D_{0} D_{1} y_{k 0} \\
& \quad-\sum_{i=1}^{N_{2}}\left(C_{i}^{k}+C_{i}^{m_{\mathrm{t}}}\right) y_{i 0} a_{0} \cos \bar{\omega} \tau \\
& \quad+\sum_{i=1}^{N_{2}} \sum_{j=1}^{N_{2}} \sum_{h=1}^{N_{2}} D_{i j h}^{k} y_{i 0} y_{j 0} y_{h 0} \\
& +\sum_{i=1}^{N_{2}} \sum_{j=1}^{N_{2}} \sum_{h=1}^{N_{2}}\left(E_{i j h}^{k}+E_{i j h}^{m_{\mathrm{t}}}\right) y_{i 0}\left(y_{j 0} D_{0}^{2} y_{h 0}\right. \\
& \left.+D_{0} y_{j 0} D_{0} y_{h 0}\right)=0,
\end{aligned}
$$

where, $D_{0}=\partial / \partial T_{0}$ and $D_{1}=\partial / \partial T_{1}$.

The general solution of Eq. (30) can be represented as:

$y_{k 0}=A_{k}\left(T_{1}\right) \exp \left(i \omega_{k} T_{0}\right)+\bar{A}_{k}\left(T_{1}\right) \exp \left(-i \omega_{k} T_{0}\right)$.

where $A_{k}$ and $\bar{A}_{k}$ are a complex conjugate functions in $T_{1}$.

Substituting Eq. (32) into Eq. (31) yields:

$$
\begin{aligned}
& D_{0}^{2} y_{k 1}+\omega_{k}^{2} y_{k 1}=-2 i \omega_{k}\left(\xi_{k} A_{k}+D_{1} A_{k}\right) \exp \left(i \omega_{k} T_{0}\right) \\
& +\frac{1}{2} \sum_{n=1}^{N_{2}}\left(C_{i}^{k}+C_{i}^{m_{\mathrm{t}}}\right)\left\{A_{n} \exp \left[i\left(\omega+\omega_{n}\right) T_{0}\right]\right. \\
& \left.\quad+\bar{A}_{n} \exp \left[i\left(\omega-\omega_{n}\right) T_{0}\right]\right\}+\sum_{n=1}^{N_{2}} \sum_{j=1}^{N_{2}} \sum_{h=1}^{N_{2}} \\
& \left\{\begin{array}{c}
{\left[-D_{n j h}^{k}+\left(\omega_{j} \omega_{h}+\omega_{h}^{2}\right)\left(E_{n j h}^{k}+E_{n j h}^{m_{\mathrm{t}}}\right)\right] \bar{A}_{n} A_{j} A_{h} \exp \left[i\left(-\omega_{n}+\omega_{j}+\omega_{h}\right) T_{0}\right]} \\
+\left[-D_{n j h}^{k}+\left(\omega_{h}^{2}-\omega_{j} \omega_{h}\right)\left(E_{n j h}^{k}+E_{n h}^{m_{\mathrm{t}}}\right)\right] A_{n} \bar{A}_{j} A_{h} \exp \left[i\left(\omega_{n}-\omega_{j}+\omega_{h}\right) T_{0}\right] \\
{\left[-D_{n j h}^{k}+\left(\omega_{h}^{2}-\omega_{j} \omega_{h}\right)\left(E_{n j h}^{k}+E_{n j h}^{m_{\mathrm{t}}}\right)\right] \bar{A}_{n} \bar{A}_{j} A_{h} \exp \left[i\left(-\omega_{n}-\omega_{j}+\omega_{h}\right) T_{0}\right]} \\
+\left[-D_{n j h}^{k}+\left(\omega_{j} \omega_{h}+\omega_{h}^{2}\right)\left(E_{n j h}^{k}+E_{n j h}^{m_{\mathrm{t}}}\right)\right] A_{n} A_{j} A_{h} \exp \left[i\left(\omega_{n}+\omega_{j}+\omega_{h}\right) T_{0}\right]
\end{array}\right]+c c,
\end{aligned}
$$

where $\bar{\Delta}$ shows the conjugate function of $\Delta$ and $c c$ represents the conjugate of preceding terms.

In order to analyze the principal parametric resonance of the $P$-order mode for the FCRM, the detuning parameter $\sigma$ is introduced as:

$\bar{\omega}=2 \omega_{p}+\varepsilon \sigma$. 

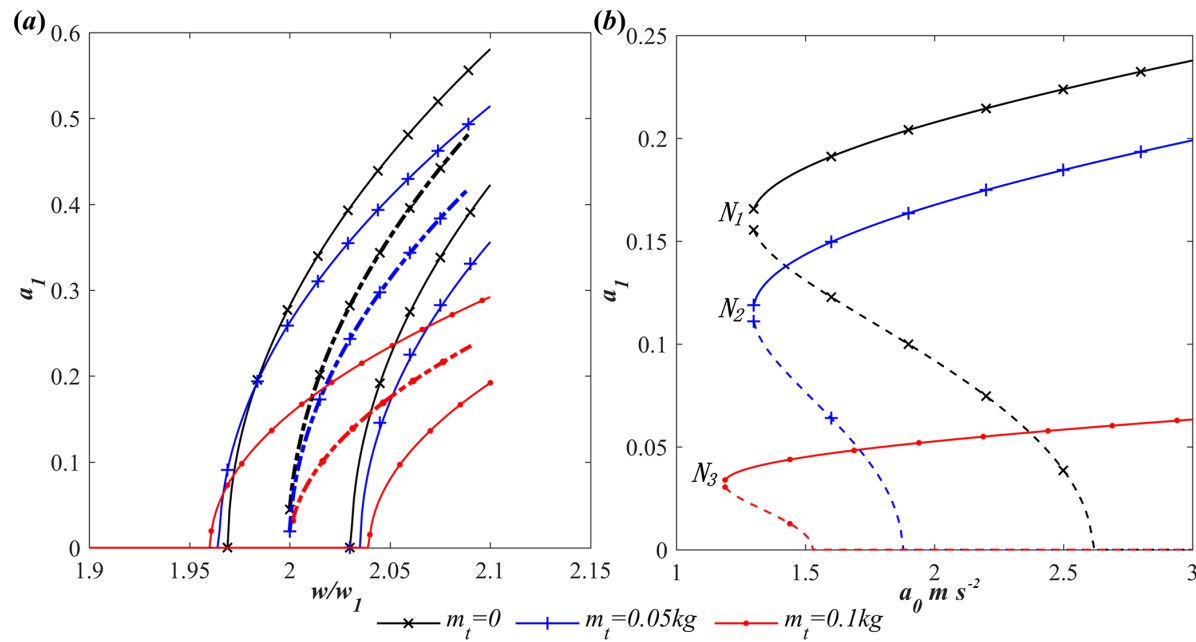

Figure 4. Steady-state response curves of the principal parameter resonance for the first-order mode of the FCRM with different terminal load: (a) amplitude frequency response curves $\left(a_{0}=1.5 \mathrm{~m} \mathrm{~s}^{-2}\right)$; (b) excitation-amplitude curves.


Figure 5. Steady-state response curves of the principal parameter resonance for the second-order mode of the FCRM with different terminal load: (a) amplitude frequency response curves $\left(a_{0}=1.5 \mathrm{~m} \mathrm{~s}^{-2}\right)$; (b) excitation-amplitude curves.

Substituting Eq. (34) into Eq. (33) and eliminating the secular terms leads to:

$$
\begin{aligned}
& 2 i \omega_{p}\left(\xi_{p} A_{p}+D_{1} A_{p}\right)-\frac{1}{2}\left(C_{p}^{p}+C_{p}^{m_{\mathrm{t}}}\right) \bar{A}_{p} \exp \left(i \sigma T_{1}\right) \\
& +\left[3 D_{P P P}^{P}-2 \omega_{P}^{2}\left(E_{P P P}^{P}+E_{P P P}^{m_{\mathrm{t}}}\right)\right] \bar{A}_{p} A_{P}^{2}=0 .
\end{aligned}
$$

Defining the form of $A_{P}$ as:

$A_{p}=\frac{1}{2} a_{p}\left(T_{1}\right) \exp \left(i \lambda_{p}\right)$

where $a_{p}$ and $\lambda_{p}$ are the real functions in $T_{1}$.
Through substituting Eq. (36) into Eq. (35) and separating of real and imaginary parts, one may obtain:

$$
\left\{\begin{aligned}
a_{p}^{\prime} & =-\xi_{p} a_{p}+\frac{1}{4 \omega_{p}}\left(C_{p}^{p}+C_{p}^{m_{\mathrm{t}}}\right) a_{p} \sin \zeta \\
a_{p} \zeta^{\prime} & =\sigma a_{p}+\left[\frac{1}{2} \omega_{p}\left(E_{P P P}^{P}+E_{P P P}^{m_{\mathrm{t}}}\right)-\frac{3 D_{P P P}^{P}}{4 \omega_{p}}\right] a_{p}^{3}, \\
& +\frac{1}{2 \omega_{p}}\left(C_{p}^{p}+C_{p}^{m_{\mathrm{t}}}\right) a_{p} \cos \zeta
\end{aligned}\right.
$$

where $\zeta=\sigma T_{1}-\lambda_{p}$.

Equation (37) is the average equation of the original system, which can fully reflect the system dynamics behavior near the balance point. For steady state response $\left(a_{p 0}, \zeta_{0}\right)$, $a_{p}^{\prime}$ and $\zeta^{\prime}$ equal to zero. Eliminating $\zeta$ from Eq. (37), one 
may obtain:

$$
\begin{aligned}
& {\left[\frac{3}{2} D_{P P P}^{P}-\omega_{P}^{2}\left(E_{P P P}^{P}+E_{P P P}^{m_{\mathrm{t}}}\right)\right]^{2} a_{p}^{6}} \\
& -4 \sigma \omega_{P}\left[\frac{3}{2} D_{P P P}^{P}-\omega_{P}^{2}\left(E_{P P P}^{P}+E_{P P P}^{m_{\mathrm{t}}}\right)\right] a_{p}^{4} \\
& +\left[16 \omega_{P}^{2} \xi_{P}^{2}+4 \sigma^{2} \omega_{P}^{2}-\left(C_{p}^{p}+C_{p}^{m_{\mathrm{t}}}\right)^{2}\right] a_{p}^{2}=0 .
\end{aligned}
$$

Equation (38) is a resonance curve equation of the response amplitude $\left(a_{p}\right)$ for the $P$-order mode of the FCRM as an implicit function of the detuning parameter $(\sigma)$, the amplitude of the base excitation $\left(a_{0}\right)$ and the terminal load $\left(m_{\mathrm{t}}\right)$.

Figures 4 and 5 show the steady-state response curves of the principal parameter resonance for the first two order modes of the FCRM with different terminal load, where the solid lines indicate the amplitude-frequency response curves of the FCRM under the impact of different terminal load and the dash-dotted lines show the spine lines of the corresponding amplitude-frequency response curves in Figs. $4 \mathrm{a}$ and $5 \mathrm{a}$. It is obtained that the amplitude-frequency characteristics of the first two order modes are hard character and soft character respectively, which is identical with the results concluded by Anderson et al. (1996). Then, the correctness of the constructed dynamic model and nonlinear response analysis of the FCRM is verified.

It is seen from Figs. $4 \mathrm{a}$ and $5 \mathrm{a}$ that the increase of terminal load causes the increase of the bending degree and the resonance field of the amplitude-frequency characteristic curve for the principal parameter resonance of the FCRM system. To the first-order mode, with the increase of terminal load, the bending degree to the right of the spine line becomes larger, which means the hard character of the amplitude frequency characteristic of the first-order mode becomes stronger, as well as the resonance field of the principal parameter resonance for the first-order mode of the FCRM becomes wider. To the second-order mode, with the increase of terminal load, the soft character of the amplitude frequency characteristic becomes stronger and the resonance field becomes wider too.

Figures $4 \mathrm{~b}$ and $5 \mathrm{~b}$ show the relationship between the axial excitation amplitude of the driving base and the response amplitude of the first two order modes of the FCRM. It is obtained that the excitation acceleration value of saddlenode bifurcation becomes smaller with the increase of terminal load for both the first two order modes of the FCRM $\left(N_{1}>N_{2}>N_{3}, N_{4}>N_{5}>N_{6}\right)$. Above all, the increase of terminal load can lead the system nonlinear dynamic response to become more complex and intensifies the instability of the FCRM.

Figure 6 shows the steady-state response curves of the principal parameter resonance for the first two order modes of the FCRM under different axial excitation of the driving base. It is seen that the spine line has no changes with the in- crease of the axial excitation acceleration, which means that the axial excitation of the driving base has no influence on the hard or soft character of the amplitude frequency characteristic of the first two order modes. However, with the increase of the axial excitation acceleration, the response amplitude and the resonance field of the principal parameter resonance becomes lager, which indicates that the instability of the FCRM is intensified dramatically.

In order to intuitively express the influence of terminal load on the parametric vibration stability of the FCRM system, the stability boundaries for the principal parametric resonance of the first two order modes of the FCRM are analyzed.

Through defining $A_{p}=\frac{1}{2}\left[u\left(T_{1}\right)-i v\left(T_{1}\right)\right] \exp \left(\frac{i \sigma T_{1}}{2}\right)$ and substituting into Eq. (35), the average equations of rectangular form can be obtained as:

$$
\left\{\begin{array}{l}
\omega_{p}\left(v^{\prime}+\xi_{p} v\right)-\frac{1}{2}\left[\sigma \omega_{p}+\frac{1}{2}\left(C_{p}^{p}+C_{p}^{m_{\mathrm{t}}}\right)\right] u \\
+\frac{1}{8}\left[3 D_{P P P}^{P}-2 \omega_{P}^{2}\left(E_{P P P}^{P}+E_{P P P}^{m_{\mathrm{t}}}\right)\right] u\left(u^{2}+v^{2}\right)=0 \\
\omega_{p}\left(u^{\prime}+\xi_{p} u\right)+\frac{1}{2}\left[\sigma \omega_{p}-\frac{1}{2}\left(C_{p}^{p}+C_{p}^{m_{\mathrm{t}}}\right)\right] v \\
-\frac{1}{8}\left[3 D_{P P P}^{P}-2 \omega_{P}^{2}\left(E_{P P P}^{P}+E_{P P P}^{m_{\mathrm{t}}}\right)\right] v\left(u^{2}+v^{2}\right)=0
\end{array} .\right.
$$

The trivial solution of Eq. (39) corresponds to the periodic solution of Eq. (35). It is obvious that the equilibrium point of Eq. (39) is the origin of coordinates $((u, v)=(0,0))$, where the Jacobi matrix can be deduced as:

$$
\begin{aligned}
& \left.J\right|_{(u, v)=(0,0)=} \\
& {\left[\begin{array}{cc}
-\xi_{p} & -\frac{1}{2 \omega_{p}}\left[\sigma \omega_{p}-\frac{1}{2}\left(C_{p}^{p}+C_{p}^{m_{\mathrm{t}}}\right)\right] \\
\frac{1}{2 \omega_{p}}\left[\sigma \omega_{p}+\frac{1}{2}\left(C_{p}^{p}+C_{p}^{m_{\mathrm{t}}}\right)\right] & -\xi_{p}
\end{array}\right] .}
\end{aligned}
$$

The characteristic equation of Eq. (40) is:

$$
\lambda^{2}+2 \xi_{p} \lambda+\xi_{p}^{2}+\frac{1}{4 \omega_{p}^{2}}\left[\sigma^{2} \omega_{p}^{2}-\frac{1}{4}\left(C_{p}^{p}+C_{p}^{m_{\mathrm{t}}}\right)^{2}\right]=0 .
$$

The existence of the positive real parts for the eigenvalues of the Jacobin matrix leads to the instability of the trivial solution of Eq. (39). According to the Routh-Hurwitz criterion, the sufficient and necessary conditions for all the roots of Eq. (41) have negative real parts are that all the RouthHurwitz determinants are greater than zero, which is shown as:

$$
\left\{\begin{array}{l}
2 \xi_{p}>0 \\
\xi_{p}^{2}+\frac{1}{4 \omega_{p}^{2}}\left[\sigma^{2} \omega_{p}^{2}-\frac{1}{4}\left(C_{p}^{p}+C_{p}^{m_{\mathrm{t}}}\right)^{2}\right]>0
\end{array} .\right.
$$

Thus, the condition for the stability of the trivial solution of Eq. (39) is:

$$
\left|a_{0}\right|<\frac{2\left[\varepsilon L\left(M_{k}+M M_{k}\right)\right] \sqrt{16 \omega_{p}^{2} \xi_{p}^{2}+4 \sigma^{2} \omega_{p}^{2}}}{\bar{m} T^{2} \widetilde{\beta}_{p p}+2 T^{2} \widetilde{C}_{p}^{p}} .
$$



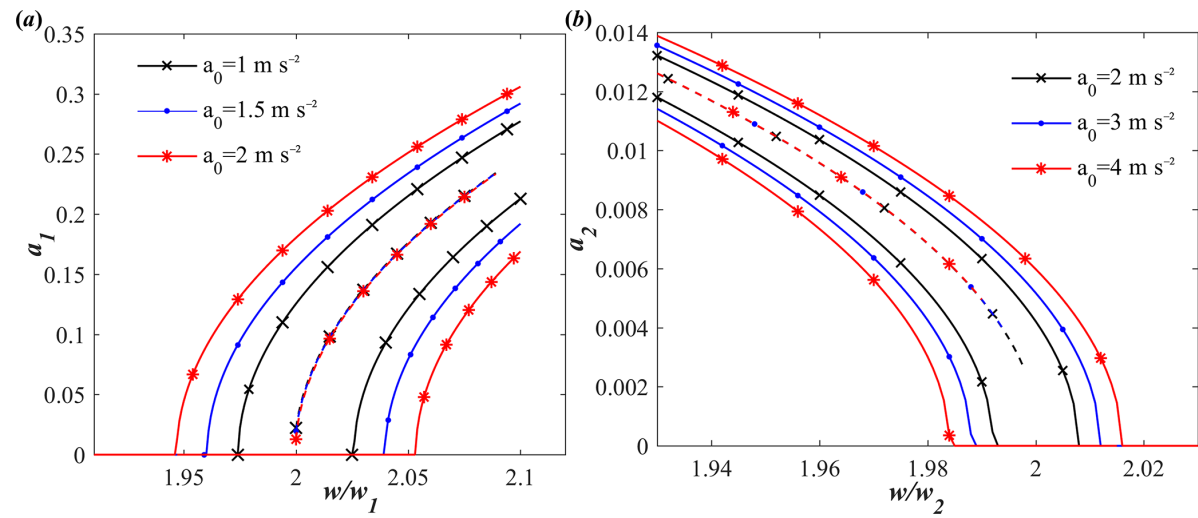

Figure 6. Steady-state response curves of the principal parameter resonance for the first two order modes of the FCRM with different base excitation: (a) amplitude frequency response curves of the first-order mode; (b) amplitude frequency response curves of the second-order mode.

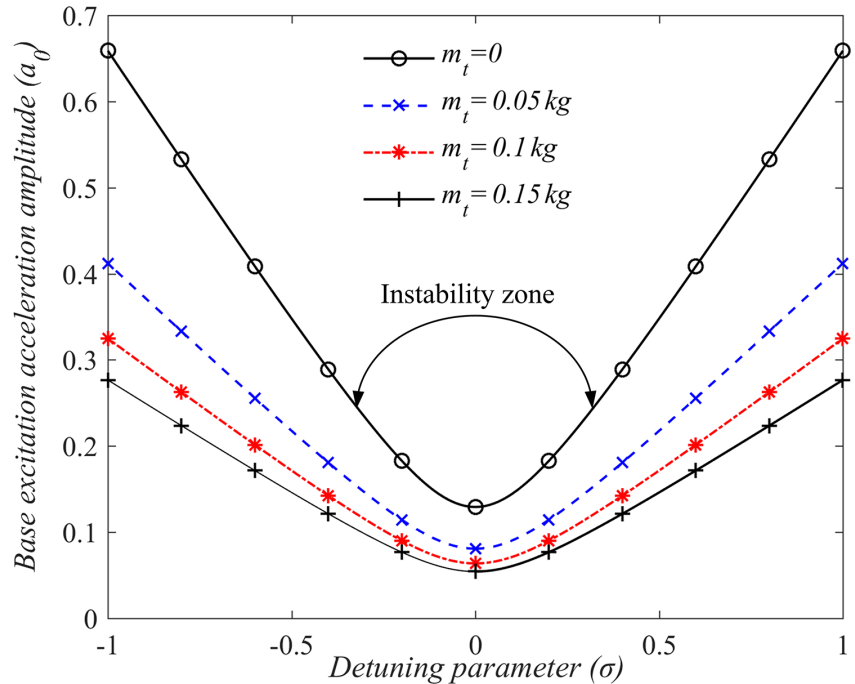

Figure 7. The stability boundaries for the principal parametric resonance of the first-order mode of the FCRM with different terminal load in $\sigma-a_{0}$.

The linear viscous damping of the first two order modes is defined as 0.1 and the range of the detuning parameter $\sigma$ is given as $[-1,1]$. Figures 7 and 8 shows the stability boundaries of the principal parametric resonance for the first-order and second-order modes of the FCRM with different terminal load, respectively. In general, for a given detuning parameter $\sigma$, the excitation amplitude of the base acceleration, causing the instability, decreases with the increase of terminal load. Furthermore, the instability zones become lager with the increase of terminal load for both the principal parametric resonance of the first-order and second-order modes, when the excitation amplitude of the base acceleration is given. Thus, in the traditional study, owing to the neglect of terminal load, the prediction for the instability boundaries of the FCRM is not safe. Above all, during the nonlinear dynamics and sta-



Figure 8. The stability boundaries for the principal parametric resonance of the second-order mode of the FCRM with different terminal load in $\sigma-a_{0}$

bility analysis of the FCRM, the impact of the driving base disturbance and the terminal load should be taken into account.

\section{Conclusions}

This paper mainly investigates the influence of base disturbance and terminal load on the nonlinear response and dynamic stability of the FCRM system. The nonlinear dynamic model of the transverse vibration for the FCRM system, considering the high-order rigid-flexible coupling terms between the large overall motion of the driving base and the elastic vibration of the flexible manipulator, is constructed. Based on the established nonlinear dynamic model and multiple scales method, the amplitude-frequency characteristics 
and parametric vibration stability of the FCRM are studied. The results demonstrate that the amplitude of the first-order mode shape decreases obviously, and the second node of the second-order mode shape as well as the third node of the third-order mode shape move away with the fixed end of the flexible manipulator, with the increase of terminal load. Furthermore, the excitation acceleration value of saddle-node bifurcation becomes smaller with the increase of terminal load for both the first two order modes of the FCRM. And the instability of the FCRM is intensified with the increase of the base excitation acceleration and terminal load, which is revealed in the increase of the resonance fields and instability areas of the principal parameter resonance of the first two order modes for the FCRM. Thus, the influence of base disturbance and terminal load should be taken into consideration during the kinetic analysis of the FCRM.

Data availability. All datasets used in the manuscript can be requested from the corresponding author Wei Li (cmeecumt512@yahoo.com). 


\section{Appendix A}

List of symbols Meanings

\begin{tabular}{ll}
\hline$m_{\mathrm{b}}$ & Mass of the base \\
$m_{\mathrm{t}}$ & Mass of the terminal operated object \\
$A$ & Cross-sectional area of the flexible manipulator \\
$L$ & Length of the flexible manipulator \\
$E I$ & Bending modulus of the flexible manipulator \\
$\rho$ & Density of the flexible manipulator \\
$\omega_{x}$ & Axial deformation of the flexible manipulator \\
$\omega_{y}$ & Transverse deformation of the flexible manipulator \\
$\omega_{u}$ & Overall longitudinal elongation of the flexible manipulator \\
$\omega_{g}$ & Coupling deformation of the flexible manipulator \\
$P$ & Axial force of the flexible manipulator \\
$M$ & Lateral bending moment of the flexible manipulator \\
$U$ & Elastic potential energy of the FCRM system \\
$T$ & Kinetic energy of the FCRM system \\
$R_{x}$ & Axial displacement of the base \\
$R_{y}$ & Lateral displacement of base \\
$\psi_{i}$ & Mode shapes functions of the longitudinal vibration \\
$Q_{i}$ & Generalized coordinates of the longitudinal vibration \\
$N_{1}$ & Mode truncation numbers of the longitudinal vibration \\
$\varphi_{i}$ & Mode shapes functions of the transverse vibration \\
$q_{i}$ & Generalized coordinates of the transverse vibration \\
$N_{2}$ & Mode truncation numbers of the transverse vibration \\
$\vartheta$ & Generalized coordinate of the second Lagrange equation \\
$F_{\vartheta}$ & Generalized force of the second Lagrange equation \\
$\tau$ & Dimensionless time constant \\
$\varepsilon$ & A book keeping parameter \\
$y_{k}$ & Approximate analytical solution of Eq. (17) \\
$y_{k}$ & First order approximation of $y_{k}$
\end{tabular}


Author contributions. WL and JYJ put forward the initial ideas; YQW guided the use of multiple scales method; MB Fan and JYJ developed the model code and performed the simulations; XFY contributed the analysis tools; JYJ prepared the manuscript with contributions from all co-authors.

Competing interests. The authors declare that they have no conflict of interest.

Acknowledgements. This work was partially supported by the National Natural Science Foundation of China (no. U1610111 and no. 51307172), the Six Talent Peaks Project in Jiangsu Province (no. ZBZZ-041), the Postgraduate Cultivation and Innovation Project of Jiangsu Province (KYZZ16-0213) and the Project Funded by the Priority Academic Program Development of Jiangsu Higher Education Institutions (PAPD)

Edited by: Ali Konuralp

Reviewed by: two anonymous referees

\section{References}

Abe, A.: Trajectory planning for flexible Cartesian robot manipulator by using artificial neural network: numerical simulation and experimental verification, Robotica, 29, 797-804, https://doi.org/10.1017/S0263574710000767, 2011.

Anderson, T. J., Nayfeh, A. H., and Balachandran, B.: Experimental verification of the importance of the nonlinear curvature in the response of a cantilever beam, ASME, J. Vib. Acoust., 118, 2127, https://doi.org/10.1115/1.2889630, 1996.

Atsumi, T., Arisaka, T., Shimizu, T., and Masuda, H.: Head-positioning control using resonant modes in hard disk drives, IEEE-ASME T. Mech., 10, 378-384, https://doi.org/10.1109/TMECH.2005.852445, 2005.

Chen, L. Q. and Yang, X. D.: Stability in parametric resonance of axially moving viscoelastic beams with time-dependent speed, J. Sound Vib., 284, 879-891, https://doi.org/10.1016/j.jsv.2004.07.024, 2005.

Chu, S. M., Cao, D. Q., Sun, S. P., Pan, J. Z., and Wang, L. G.: Impact vibration characteristics of a shrouded blade with asymmetric gaps under wake flow excitations, Nonlinear Dynam., 72, 539-554, https://doi.org/10.1007/s11071-012-0732-4, 2013.

Coleman, M. P. and Mcsweeney, L. A.: Analysis and computation of the vibration spectrum of the cartesian flexible manipulator, J. Sound Vib., 274, 445-454, https://doi.org/10.1016/j.jsv.2003.09.036, 2004.

De Luca, A. and Siciliano, B.: Closed-form dynamic model of planar multilink lightweight robots, IEEE T. Syst. Man Cy., 21, 826-839, https://doi.org/10.1109/21.108300, 1991.

Duc, N. D., Cong, P. H., and Quang, V. D.: Nonlinear dynamic and vibration analysis of piezoelectric eccentrically stiffened FGM plates in thermal environment, Int. J. Mech. Sci., 115/116, 711722, https://doi.org/10.1016/j.ijmecsci.2016.07.010, 2016.

Feliu, V., Pereira, E., and Díaz, I. M.: Passivity-based control of single-link flexible manipulators using a lin- ear strain feedback, Mech. Mach. Theory, 71, 191-208, https://doi.org/10.1016/j.mechmachtheory.2013.07.009, 2014.

Feng, Z. H. and Hu, H. Y.: Largest Lyapunov Exponent and almost Certain Stability Analysis of Slender Beams Under a Large Linear Motion of Basement Subject to Narrowband Parametric Excitation, J. Sound Vib., 257, 733-752, https://doi.org/10.1006/jsvi.2002.5041, 2002.

Huang, Y. and Li, X. F.: A new approach for free vibration of axially functionally graded beams with nonuniform cross-section, J. Sound Vib., 329, 2291-2303, https://doi.org/10.1016/j.jsv.2009.12.029, 2010.

Kiang, C. T., Spowage, A., and Yoong, C. K.: Review of control and sensor system of flexible manipulator, J. Intell. Robot. Syst., 77, 187-213, https://doi.org/10.1007/s10846-014-0071-4, 2015.

Liu, Y. F., Li, W., Yang, X. F., Fan, M. B., Wang, Y. Q., and Lu, E.: Vibration Response and Power Flow Characteristics of a Flexible Manipulator with a Moving Base, Shock Vib., 2015, 1-8, https://doi.org/10.1155/2015/589507, 2015.

Mann, M. P., Rubinstein, D., and Shmulevich, I., Linker, R., and Zion, B.: Motion planning of a mobile Cartesian manipulator for optimal harvesting of 2-d crops, T. ASABE, 57, 283-295, https://doi.org/10.13031/trans.57.10225, 2014.

Neto, M. A., Ambrósio, J. A. C., and Roseiro, L. M.: Active vibration control of spatial flexible multibody systems, Multibody Syst. Dyn., 30, 1-23, https://doi.org/10.1007/s11044-013-93413, 2013

Özkaya, E. and Pakdemirli, M.: Group-theoretic approach to axially accelerating beam problem, Acta Mech., 155, 111-123, https://doi.org/10.1007/BF01170843, 2002.

Park, C. H., Park D. I., and Park, J. H.: Vibration control of flexible mode for a beam-type substrate transport robot, Int. J. Adv. Robot. Syst., 10, 1-7, https://doi.org/10.5772/56752, 2013.

Pratiher, B. and Bhowmick, S.: Nonlinear dynamic analysis of a Cartesian manipulator carrying an end effector placed at an intermediate position, Nonlinear Dynam., 69, 539-553, https://doi.org/10.1007/s11071-011-0285-y, 2011.

Qian, S., Zi, B., and Ding, H.: Dynamics and trajectory tracking control of cooperative multiple mobile cranes, Nonlinear Dynam., 83, 89-108, https://doi.org/10.1007/s11071-015-2313-9, 2016.

Qiu, Z. C.: Adaptive nonlinear vibration control of a Cartesian flexible manipulator driven by a ballscrew mechanism, Mech. Syst. Sig. Process., 30, 248-266, https://doi.org/10.1016/j.ymssp.2012.01.002, 2012.

Singiresu, S. R.: Mechanical Vibration, 4th Edn., Pearson Education, Upper Saddle River, NJ, USA, 2004.

Srinivasan, H., Harrysson, O. L. A., and Wysk R. A.: Automatic part localization in a CNC machine coordinate system by means of 3D scans, Int. J. Adv. Manuf. Tech., 81, 1127-1138, https://doi.org/10.1007/s00170-015-7178-z, 2015.

$\mathrm{Wu}, \mathrm{F}$. and Zhang, X.: An inspection and classification method for chip solder joints using color grads and Boolean rules, Robot. Cim-Int. Manuf., 30, 517-526, https://doi.org/10.1016/j.rcim.2014.03.003, 2014.

Yan, T., Yang, J., and Kitipornchai, S.: Nonlinear dynamic response of an edge-cracked functionally graded Timoshenko beam under parametric excitation, Nonlinear Dynam., 67, 527540, https://doi.org/10.1007/s11071-011-0003-9, 2012. 
Zhang, D. J., Liu, C. Q., and Huston, R. L.: On the Dynamics of an Arbitrary Flexible Body with Large Overall Motion: An Integrated Approach, Mech. Based Des. Struc., 23, 419-438, https://doi.org/10.1080/08905459508905245, 1995.
Zi, B. and Zhou, B. A.: Modified hybrid uncertain analysis method for dynamic response field of the LSOAAC with random and interval parameters, J. Sound Vib., 374, 111-137, https://doi.org/10.1016/j.jsv.2016.03.032, 2016. 